\title{
Summaries of SpS17 Discussions IAU GA 2012 Special Session on Light Pollution
}

\author{
Constance E. Walker ${ }^{1}$, Bob Parks ${ }^{2}$, Dan McKenna ${ }^{3}$, \\ Ramotholo Sefako ${ }^{4}$, Malcolm Smith ${ }^{5}$ and David Galadí-Enríquez ${ }^{6}$ \\ ${ }^{1}$ National Optical Astronomy Observatory, Tucson, AZ, USA, cwalker@noao.edu \\ ${ }^{2}$ International Dark-Sky Association, USA, bparks@darksky .org \\ ${ }^{3}$ Palomar Observatory, PO Box 200, Palomar Mountain, CA 92060, USA, \\ dmck@astro.caltech.edu \\ ${ }^{4}$ South African Astronomical Observatory, PO Box 9, Observatory, 7935, South Africa, \\ rrs@saao.ac.za \\ ${ }^{5}$ Cerro Tololo Interamerican Observatory, Casilla 603, La Serena, Chile, \\ msmith@ctio.noao.edu \\ ${ }^{6}$ Centro Astronómico Hispano-Alemán (CAHA), Apartado 2010, ES-04080-Almería, Spain, \\ email: dgaladi@caha.es
}

\section{Introduction}

To address light pollution issues, IAU Commissions 41, 46, 50, and 55 are involved in getting the word out to the public and IAU members via cultural, educational, technical; however, efforts can always improve and evolve. To carry out a successful light pollution abatement program supported by the IAU, it takes a diversity of groups, professions, and disciplines with their collective knowledge and experience. In manifesting dark skies awareness effectively, we are stronger together than we are alone; therefore, combining efforts of Commissions 41, 46, 50 and 55 with organizations like the International DarkSky Association, Astronomers Without Borders, The World at Night and partnering with events like Earth Hour or GLOBE at Night is a good step forward.

\section{Light Pollution and the Public}

- The Role of Media, Planetaria and Amateur Astronomers in Light Pollution Education

- Light Pollution Education in Schools and in Cultures

- Global Star-Hunting and "Nights of Darkness" Campaigns

The presentations and discussion on September 29 for the IAU GA 2012s Special Session on Light Pollution, SpS17, focused on the role of planetaria, amateur astronomy associations and media to change perspective, global initiatives in light pollution education, and global campaigns on dark skies awareness. In the discussion at the end of the day, a few key points and actions were identified, hopefully as items IAU members (collectively and individually) can support:

(a) Teach through the students with well-trained educators:

It is our responsibility to influence and inspire the next generation, since it is on their shoulders to make a difference in society. If they feel convinced something is important, they will act to change society. This process is accomplished in part by means of teachers we train. More light pollution education can be done through the IAUs GTTP, NASE 
and UNAWE programs through the professional development for teachers and learning experiences for students.

(b) Find a way to reach the public through the media and global events:

If you have never seen the Milky Way Galaxy, you are less inclined to invest in a problem that you are not convinced exists. Until you can get most people aware there is a problem, then the problem cannot be changed. We (IAU) have to have a better way to break into the news media. The media generates a consensus among the people to push the government to do something. We need to package light pollution (as sound bites) in terms of its relation to many different types of issues (the environment, health, air pollution, climate change, etc), so that the news media is interested and can easily write about it. One suggestion was to start a campaign similar to past successful campaigns demoting cigarette smoking and littering or to collaborate with the organizers of a successful campaign like Earth Hour, for example. For an easy recommendation, more people could include light pollution issues in their oral presentations and in planetarium shows. Losing the Dark is a 5-minute planetarium public service announcement that is free to all planetaria that will help accomplish this task. Also, astronomy is easier to visualize and thereby appreciate than other sciences. By offering more night sky images to the news media, people can see for themselves what they are missing (e.g., The World at Night and its annual Earth and Sky Photo Contest).

With the public in various countries, astro-tourism is on the rise and is another excellent venue for raising dark-skies awareness. This topic is addressed in a later session of the IAU GA 2012 SpS17 on Light Pollution.

(c) Acquire more data to state factually what the numbers are:

Numbers support why steps need to be taken to redress light pollution issues. Raising awareness is good but the bottom line is that we need to measure whether we are protecting the night sky. Documenting the degradation of the night sky or its improvement is important, in addition to improving legislation. Numbers for instance would support why we should conserve energy and how that affects cost. Other session participants felt it is the sense of wonder not wallets.

In some areas there is not yet a critical awareness that excites people to do enough research to back up the effects of light pollution on things like crime levels or the increased use of blue lights. We better be ready to address proactively these issues the day after we have promoted our stance in the media.

(d) Convince authorities through IAU:

If, for instance, the IAU can address the European Parliament, and as a community, we use this as momentum to organize action around the world, this may prove an effective means to an end. Officers of Commissions 46 and 50 (on Education and Observatory Site Protection) were present at the IAU GA 2012 Special Session on Light Pollution, SpS17. In the next three years they hope to carry these ideas forward by working together with Commissions 41 and 55 (on World Heritage and Communicating with the Public) and the participants of SpS17 and other identified stakeholders in a variety of areas (health, environment, engineering, astronomy, etc) and from a variety of organizations (IDA, Astronomers Without Borders, CIE, etc). Not only does the topic of light pollution touch upon different areas, but people involved in these different areas are stronger working together than separately. 


\section{Social Event at the Beijing Planetarium (Premier of Losing the Dark)}

The IAU SpS17 hosted an evening program focused on light pollution and night sky protection at the Beijing Planetarium. There were about 100 people in attendance, on Wednesday August 29th. The event was informative, well attended and gave attendees an opportunity to interact in a casual environment.

The session began with an award ceremony for Dr. Malcolm Smith, from the National Optical Astronomy Observatory (NOAO), He was honored for his substantial contributions to light pollution abatement on behalf of astronomical observatories and the community at large. Dr. David Silva (NOAO Director) and Bob Parks (International DarkSky Association Executive Director) presented Dr. Smith with the Dr. David Crawford Lifetime Achievement Award. This award, in honor of the International Dark-Sky Associations (IDA) co-founder and first executive director, recognizes those who have made a sustained effort to raise awareness of light pollution and to help implement solutions to light pollution.

After the award ceremony, the International Dark-Sky Association previewed a fulldome planetarium show entitled Losing the Night. This short educational program explains in simple terms what light pollution is, its impact on the environment and how it can be reduced. It was produced by Loch Ness Productions for IDA and is intended to be shown as a preview to regular planetarium shows. It is hoped that this program will help raise international awareness of issues related to light pollution and reach new audiences. It will be available for free to planetariums and a flat screen version will also be available for educational purposes.

After the showing of Losing the Dark, the attendees were treated to a showing of the documentary film The City Dark. The City Dark is an award-winning film documentary, which was directed by independent filmmaker Ian Cheney. It documents his experience of moving to New York City from rural Maine. The film covers a wide range of issues related to light pollution in a very accessible fashion. Mr. Cheney has interviewed individuals with unique perspectives on how the loss of the night sky impacts society and the environment.

\section{Dark Sky Places, Starlight Reserves and Astro-Tourism}

The effort to promote the preservation of Dark Skies has the greatest appeal in numbers based on emotional, rather than scientific needs. Astro-tourism spans all cultures that have different views of the night sky as well as varied context defined by history, geography and climate.

The interests in promoting tourism in general can be at odds with astro-tourism due to the lighting of architectural features and public areas. Solutions to optimize the partnership with astro-tourism will not be universal, but on individual bases. Two strategies seem to emerge: create the largest area to protect, such as the U.S National Park Service and identifying small, close-by viewing areas. One such solution on the island of Malta is to provide night sky tours by boat.

Promotion of dark-sky awareness has a day-time component that should not be overlooked. Parks that have adopted and developed astronomy-based themes such as solar system scaled trails with sponsored markers, sundials, or gnomon provide activities that will naturally lead into night-time sky awareness. 


\section{Dark Skies Measurements and Site Monitoring}

The IDA has been promoting dark-sky awareness and advocating the protection of the night sky for over twenty years. An estimation that $75 \%$ of the current population of the Earth has never seen the Milky Way leads to the thought that if people do not know what they have lost, it is hard to convince them to get it back. The IDA and the IAU could develop a stronger partnership that would strengthen both organizations. The IDA is active in park certification, promoting research, developing technical standards, informing on solid-state lighting technology, supporting legislation, monitoring of night sky brightness, and has a focus on youth. The IDA emphasized that this developed expertise could be of great value to the IAU commission 50 .

Measurements of light sources from air and spacecraft contribute valuable data for monitoring and modeling the characteristics of ground-based light sources. Measurements by aircraft can provide highly calibrated measurements of the low angle scattering, the elevation, azimuth, and spatial as well as spectral distribution. Measurements from the International Space Station using digital cameras that record color images in the RAW mode have been found to be very linear and provide high-quality data when combined with aircraft ground truth. The ISS data combines calibration from ground truth and stellar background fields.

The need for accurate long-term night sky brightness measurements is critical and urgent. Even with well-calibrated instrumentation, a change in sky brightness due to the atmosphere or light pollution is difficult to untangle. As time progresses the technology used to measure night sky brightness will evolve. It is therefore necessary to adopt standards in reporting measurements that will provide the necessary information to unify datasets. Presently there are two types of night sky photometers used to collect data: the Sky Quality Meter (SQM) family manufactured by Unihedron and the Night Sky Brightness Monitor (NSBM) built by Matrix Development under contract from the SKYMONITOR project. Both devices use the same optical filter and detector and thus have the same spectral response. The NSBM has a narrower field of view, about 4 degrees with a baffle tube for nearby light rejection. SQMs are producing the majority of the new, sky brightness, data and it is clear that the calibration must be understood both in terms of short and long-term stability.

\section{Light Pollution Legislation and Protecting Observatory Sites}

There is a need to protect the night sky against light pollution as both a heritage of citizens and a scientific resource. Low-pressure sodium (LPS) is recommended as an efficient form of lighting that is less detrimental to astronomy, and has potential for energy savings. It is important to consider impacts that light pollution would cause to the environment and astronomy, but also consider the potential benefits of using efficient lighting or using lighting only when necessary.

A question was asked about how do we help change the culture of the lighting industry to be more sensitive to light pollution issues?

The following were noted:

- to recognize the need for light pollution model

- to recognize the need for one model of legislation regarding lighting

- to recognize that light pollution increases every day and

- to recognize the need for concrete results that everyone is likely to believe

Different groups of people are concerned with light pollution; however, we as a group are not necessarily (as with astronomy) the main role player. We should therefore not 
compromise without consulting other role players and we should be aware of the importance of the concerns of the general populous regarding light pollution. We should consider using the IAUs status as a large organization to influence governments decisions on lighting legislation. We should also support our statements with factual statistics and numbers that make sense and that are acceptable when discussing the effects of light pollution on human beings and other animals.

Given that scientists don't write laws, but engineers do have a hand in standardizing lighting regulations, we need to make sure that lighting industries and lighting engineers are closer to reality and that contradictions with what astronomers want are minimized.

There is a need for understanding the technical aspects of lighting as defined by the CIE. Focus should shift towards use of lighting, not dark sky. We should only use the night sky as a reference point. It should be pointed out that in the strictest sense, all light is bad, and LEDs are worse and do not beat High-Pressure Sodium and Low-Pressure Sodium lights when it comes to efficiency.

\section{Progress and Action Plan for Implementing IAU 2009 Resolution B5}

This subsession began with a distribution of copies of B5. The resolution can be located at: http ://www.iau.org/static/resolutions/IAU2009_English.pdf

Consideration 4 urges that "IAU members be encouraged to take all necessary measures to involve the parties related to skyscape protection in raising public awareness - be it at local, regional, national, or international levels - about the contents and objectives of the International Conference in Defence of the Quality of the Night Sky and the Right to Observe Stars (http://www.starlight2007.net/), in particular the educational, scientific, cultural, health and recreational importance of preserving access to an unpolluted night sky for all humankind.

Led by the IAC, the "Starlight" Initiative and the IDA, astronomers, biologists, medical researchers, lawyers, school teachers and tourism organizations around the world have started a significant, joint, international effort to work with each other. This effort gained significant momentum during 2009 - the International Year of Astronomy - momentum that has largely been maintained since then.

Key components include:

- the education of the public, with special emphasis on working with school teachers

- the education of authorities at local, regional, national and international levels with a view to improving and extending appropriate legislation and enforcement

- alerting lighting engineers of the need to direct light where it is needed, when it is needed as well as the especially damaging impact of blue-rich light sources. A further key effort is work with UNESCO, ICOMOS, UCN and others on protection of the heritage of the natural night sky. Here the IAU's institutional support is proving particularly influential.

\section{Spectra of Artificial Blue-Rich Sources}

A dialogue was raised on which lamp should be recommended from an astronomical point of view. Amber LED, spectrally preferable, is less efficient, but there is the risk of judging tomorrow's technology according to today's standards. Both from the energetic and the spectral sides, Low-Pressure Sodium light seems the obvious choice. A balance among environmental impact and efficiency seems necessary. Taking into account other 
advantages of LED lights (they can be regulated and switched on/off rapidly), amber LEDs are not to be discarded.

Yellow light is better: it is less Rayleigh-scattered and human scotopic vision is much less sensitive to a residual yellowish skyglow.

The question is made whether not having requested full-cutoff at Hawaii from the very beginning can be considered a mistake in retrospect. The answer underlines the difficulty of negotiating under pressure and the lack of realistic choices at the time.

\section{Astronomical Input to Lighting Industry Development: Prospects for Success}

It is said that industry states cool LEDs are good for the environment, but this does not tell the entire story. The industry often does not even seem to be aware of the environmental impact of blue light (e.g., Rayleigh scattering and chronodisruption are less familiar to them).

The question was raised: Are we ready, as the IAU, to propose any specific recommendation or resolution addressed to industry?

The opinions given may be summarized: 'The narrower the band, and the redder the spectrum, the better'. Bluish light affects all of the nighttime environment, but only humans determine whether or not to put this light into the night; however, it is not clear if the IAU should dare to enter into biological or medical argumentations. Some doubts on the biological universality of blue light as a melatonin inhibitor were posed but it was stated that there are studies showing that this universality holds.

The case was made that general, but solid, physical and astronomical arguments defend a recommendation to say no to broad spectra and to blue-rich light. Then society will make a decision based on a compromise among all aspects: energy, health, biology, astronomy, but our task as astronomers is to make the case for everyone's sky.

Finally the moderator (Green) assumed the task to produce a draft based on these elementary principles: yellower, narrower. 